\title{
Operative Evaluation Of Cardiac Tamponade In A Patient With Multiple Myeloma
}

\author{
N Barbetakis, T Bishiniotis, R Valeri, M Vassiliadis, S Anisoglou, T Antoniadis, \\ C Tsilikas
}

\section{Citation}

N Barbetakis, T Bishiniotis, R Valeri, M Vassiliadis, S Anisoglou, T Antoniadis, C Tsilikas. Operative Evaluation Of Cardiac Tamponade In A Patient With Multiple Myeloma. The Internet Journal of Thoracic and Cardiovascular Surgery. 2002 Volume 5 Number 2.

DOI: $\underline{10.5580 / 1 \mathrm{de} 9}$

\begin{abstract}
Multiple myeloma presents with various clinical manifestations depending on the mode and the extent of organ involvement. Pericardial myeloma involvement and subsequent cardiac tamponade is extremely rare $(<0,2 \%)[1]$. We report the case of a patient with multiple myeloma who presented with cardiac tamponade and was evaluated surgically with thoracotomy and minimal debulking pericardiectomy (fenestration).
\end{abstract}

\section{CASE REPORT}

A 59-year-old man with an IgG multiple myeloma was admitted to our department with a 5-day history of dyspnea, cough, chest pain, fatigue and lower extremity edema. Physical examination revealed a blood pressure of 95/60 mm $\mathrm{Hg}$, tachypnea, orthopnea, jugular venous distension, distant heart sounds and decreased breath sounds at both lung bases. Admission chest $\mathrm{x}$-ray film showed a large cardiac silhouette without pulmonary congestion. The electrocardiogram (ECG) showed sinus tachycardia with low voltage throughout. ECG and cardiac enzymes showed no evidence of myocardial damage. An emergency echocardiogram demonstrated a large pericardial effusion with right atrial and right ventricular diastolic compression and an echogenic pattern of infiltrative pericarditis (Figure 1).

\section{Figure 1}

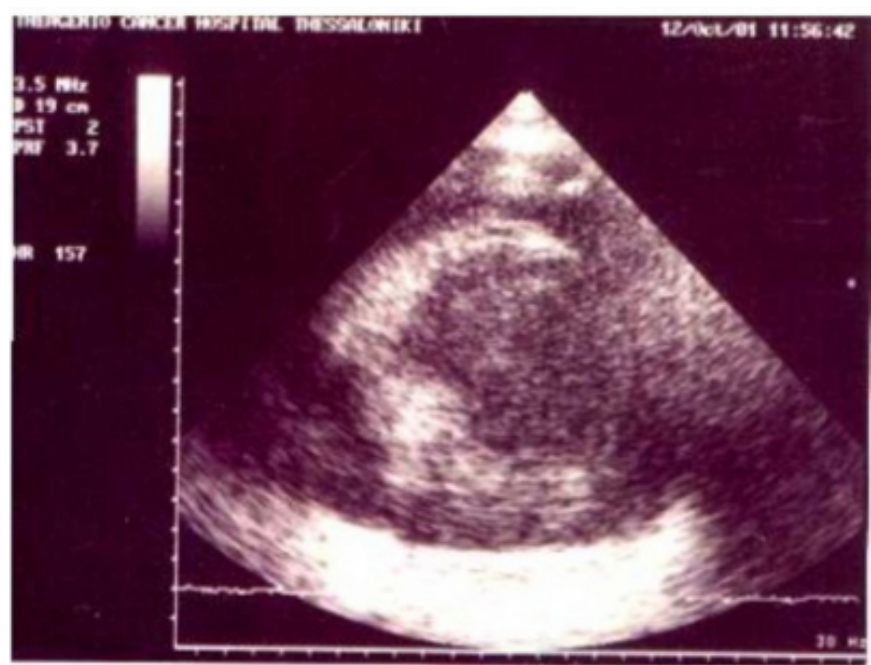

A CT scan of the chest revealed a significant amount of pericardial effusion with no evidence of superior vena cava obstruction. A diagnostic right heart catheterization revealed a central venous pres-sure of $19 \mathrm{~cm} \mathrm{H}_{2} \mathrm{O}$, a right ventricular pressure of $38 / 22 \mathrm{~mm} \mathrm{Hg}$ and a pulmonary artery pressure of $20 \mathrm{~mm} \mathrm{Hg}$. Other investigations demonstrated normochromic, normocytic anaemia (Hb: 8,4 g/dl - Ht: $25,5 \%$ ) with peripheral blood film showing occasional plasmacytoid lymphocytes. A diagnosis of cardiac tamponade was established. Pericardiocentecis was 
performed and only $5 \mathrm{ml}$ of serosanguinous pericardial fluid were aspirated. Pericardial fluid cytology was positive for malignant plasma cells (Figure 2).

\section{Figure 2}

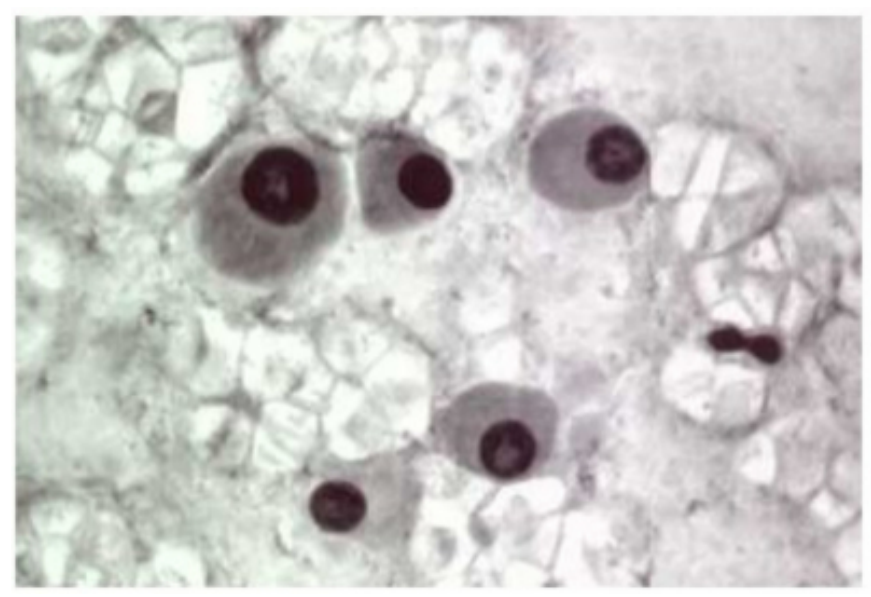

After three failed attempts at pericardiocentesis, surgical intervention (left posterolateral thoracotomy through $6^{\text {th }}$ intercostal space) with the creation of a pericardial fenestration was performed. Loculated serosanguinous pericardial effusion with fibrinous adhesions between the parietal and visceral pericardial membranes was noted and five hundred $\mathrm{ml}$ of pericardial fluid were aspirated. The patient tolerated the surgical procedure well. He underwent three echocardiograms during the postoperative period which revealed no recurrent pericardial effusion. Significant clinical improvement was noted within 3 days of pericardial fenestration formation and the patient was asymptomatic 12 days later. He was discharged home in a much improved condition. The patient died four months later of uncontrolled systemic myeloma without recurrence of pericardial effusion or constrictive physiology on repeated echocardiograms.

\section{DISCUSSION}

The majority of patients with pericardial metastases are clinically asymptomatic. In patients presenting with cardiac tamponade, however, malignancy is a common etiologic factor. In most patients urgent subxiphoid pericardiocentesis and placement of a pericardial catheter relieves tamponade. It is generally agreed that surgical intervention should be considered in those medically fit patients who-se effusive pericarditis is unresponsive to radiotherapy or to intrapericardial therapy or requiring repeated pericardiocenteses. Finally, patients with constrictive pericarditis caused by radiation or neoplastic pericardial constriction and who would be expected to have a reasonable chance of long-term survival, should have pericardial resection.

Infiltration of the pericardium by malignant cells is a common autopsy finding in patients with metastatic cancer. Malignant neoplasms of the heart and pericardium are usually the result of metastatic disease, most commonly from a primary tumor in the breast or the lung. Pericardial involvement in patients with multiple myeloma is rare.

Less than ten cases of multiple myeloma involving the pericardium have been described since 1966, with many of these cases being diagnosed at autopsy [ $\left.{ }_{2}\right]$. Of the cases reported of pericardial effusion caused by multiple myeloma, three cases were associated with cardiac tamponade. In one case by Goldberg et al.[3], the patient was admitted with congestive heart failure and a diagnosis of multiple myeloma was also made. She was discharged but returned 4 months later with severe shortness of breath and chest pain. Autopsy showed a pericardial effusion $(750 \mathrm{ml})$ with infiltration of pericardium and myocardium. In a second case, reported by Garett et al.[4], a patient with multiple myeloma presented with cardiac tamponade which was confirmed at cardiac catheterization. A pericardial biopsy revealed plasma infiltrates.

The third case, reported by Santana et al.[5], demonstrated epicardial and pericardial involvement in a patient with multiple myeloma resulting in cardiac tamponade and after a pericardial window, in constrictive pericarditis. Radiation therapy (24Gy in 15 fractions) and prednisone diminished dramatically his right heart congestive signs. This patient remained well until three months later when he died of fulminating pneumococcal pneumonia.

Our case demonstrates pericardial involvement and cardiac tamponade in a patient with multiple myeloma. Pericardiocentesis was successful but only $5 \mathrm{ml}$ of serosanguinous fluid could only be aspirated due to fibrinous adhesions between the parietal and visceral pericardial membranes and could not alleviate symptoms of tamponade. A left posterolateral thoracotomy through $6^{\text {th }}$ intercostal space and a pericardial window were performed. The patient died four months later of systemic plasmacytoma without clinical or echocardiographic evidence of recurrent pericardial effusion.

\section{CONCLUSIONS}

In conclusion, our case demonstrates the possibility of cardiac tamponade in patients with diagnosed multiple 
myeloma. We favor the operative evaluation of these patients because the debulking of infiltrated pericardium can alleviate constrictive physiology and serves as the best method to evaluate the extent of the intrathoracic primary disease.

\section{CORRESPONDENCE TO}

N. Barbetakis, MD, Resident in Cardiothoracic Surgery Samou 3, GR-54638, Thessaloniki Greece. E-mail: nibarb@otenet.gr Tel No: 0030-310216560

\section{References}

1. Rosenbaum H, Hoffman R, Carter A et al: Multiple myeloma with pericardial involvement and cardiac tamponade: A report of three patients. Leuk Lymphoma 1996;24:183-186.

2. Mitchell M, Horneffer M, Standiford T: Multiple myeloma complicated by restrictive cardiomyopathy and cardiac tamponade. Chest 1993;103:946-947.

3. Garett TJ, McCans JL, Parker JO. Fatal involvement of the heart with multiple myeloma.Can Med Assoc J 1972;107:979-980.

4. Goldberg E, Mori K. Multiple myeloma with isolated visceral (epicardial involvement) and cardiac tamponade. Chest 1970;57:584-587.

5. Santana O, Vivas P, Ramos A, Safirstein S, Agatston A. Multiple myeloma involving the pericardium associated with cardiac tamponade and constrictive pericarditis. Am Heart J 1993; 126:737-740. 


\section{Author Information}

Nikolaos Barbetakis, MD

Department of Thoracic Surgery, Theagenion Cancer Hospital

\section{Theodoros Bishiniotis, MD, PhD}

Department of Cardiology, Theagenion Cancer Hospital

\section{Rosalia Valeri, MD}

Department of Pathology, Theagenion Cancer Hospital

\section{Michalis Vassiliadis, MD}

Intensive Care Unit, Theagenion Cancer Hospital

\section{Souzana Anisoglou, MD}

Intensive Care Unit, Theagenion Cancer Hospital

\section{Theodoros Antoniadis, MD}

Department of Thoracic Surgery, Theagenion Cancer Hospital

Christodoulos Tsilikas, MD, PhD

Department of Thoracic Surgery, Theagenion Cancer Hospital 